\title{
A Spectral Solenoidal-Galerkin Method for Rotating Thermal Convection between Rigid Plates
}

\author{
Cihan Yıldırım, ${ }^{1}$ Durmuş Yarımpabuç, ${ }^{2}$ and Hakan I. Tarman ${ }^{3}$ \\ ${ }^{1}$ Mechanical Engineering Department, Akdeniz University, Antalya 07058, Turkey \\ ${ }^{2}$ Mathematics Department, Osmaniye Korkut Ata University, Osmaniye 80000, Turkey \\ ${ }^{3}$ Engineering Sciences Department, Middle East Technical University, Ankara 06531, Turkey \\ Correspondence should be addressed to Cihan Yıldırım; ycihan@akdeniz.edu.tr
}

Received 18 January 2013; Accepted 21 February 2013

Academic Editor: Safa Bozkurt Coskun

Copyright (C) 2013 Cihan Yıldirım et al. This is an open access article distributed under the Creative Commons Attribution License, which permits unrestricted use, distribution, and reproduction in any medium, provided the original work is properly cited.

\begin{abstract}
The problem of thermal convection between rotating rigid plates under the influence of gravity is treated numerically. The approach uses solenoidal basis functions and their duals which are divergence free. The representation in terms of the solenoidal bases provides ease in the implementation by a reduction in the number of dependent variables and equations. A Galerkin procedure onto the dual solenoidal bases is utilized in order to reduce the governing system of partial differential equations to a system of ordinary differential equations for subsequent parametric study. The Galerkin procedure results in the elimination of the pressure and is facilitated by the use of Fourier-Legendre spectral representation. Numerical experiments on the linear stability of rotating thermal convection and nonlinear simulations are performed and satisfactorily compared with the literature.
\end{abstract}

\section{Introduction}

Thermal convection in a fluid layer has been a cradle of nonlinear hydrodynamic stability studies. In particular, the classical Rayleigh-Bénard problem of thermal convection in a horizontal layer which is heated from below is the most studied problem of the convective flows. This is due to its stability behavior exhibiting a sequence of discrete steps from steady regime to periodic, quasiperiodic regimes and eventually to chaotic regime as well as the simplicity of its geometry. This geometry of infinite fluid layer confined between rigid plates has been approximated by a periodic horizontal extent in the numerical studies and by large-aspect-ratio containers in the experiments. The underlying nonlinear stability mechanism of transition has been extensively studied both experimentally [1] and numerically [2-4]. Rayleigh and Prandtl numbers as well as the wavelength of the imposed disturbances play important roles as control parameters in the sequence of transitions. Other control mechanisms studied may include externally imposed magnetic field and rotation [5].
In this work, the effect of rotation on thermal convection is studied using a direct numerical simulation. Early studies on this topic go back to the theoretical investigations on the linear stability with/without rotation by Chandrasekhar [5]. Another detailed linear stability analysis of rotating RayleighBénard flow was performed by Clever and Busse [6] for critical wavenumber and corresponding critical Rayleigh number values for a range of Coriolis parameter $(\Omega)$ values in a geometry of rigid plates rotating around its vertical axis. Some quantitative stability criterion is obtained depending on the varied rotation, and a stability diagram of the natural convection with respect to rotation is constructed. It is observed that coriolis force reduces the heat transport for low Rayleigh and high Prandtl number flows, while limited rotation enhances the heat transport for decreasing Prandtl number.

Early nonlinear studies include Veronis' work [7] who worked on the two-dimensional system with stress-free boundary conditions. It was observed that finite amplitude instability occurs due to the nonlinear effects and that it can be damped by increasing rotation. Further, Veronis [8] theoretically examined the effects of rotation and viscosity on 
the cellular motion of convection for different boundary conditions. A clear explanation was provided on the energy releasing and dissipative mechanisms with regards to viscosity. The effects of rotation on convection in low and high Prandtl fluids confined between stress-free boundaries were also studied by Veronis [9]. A unique feature is observed to occur that steady finite amplitude convection can exist for Rayleigh number which is lower than the critical Rayleigh number for a limited range of rotation.

Theoretical studies include those of Küppers and Lortz [10] that investigated the stability behavior for the case of infinite Prandtl number and free-free boundaries. It was shown that there is no stable steady-state convection for Taylor number which is higher than the critical Taylor number, $\mathrm{Ta}=2285(\Omega \approx 23.9)$. Küppers [11] extended the work to rigid boundaries and finite Prandtl numbers. Recently Clever and Busse [12] studied numerically two- and three-dimensional convections under the influence of rotation. Unusual dynamical features for low Prandtl number flow were the main attention in this work. Kurt et al. $[13,14]$ studied rotating cylindrical annulus with small gap approximation. They conducted stability analysis of convection influenced by rotation and magnetic field. They also observed some instabilities.

Experimentally, Rossby [15] investigated the natural convection in several fluids confined between rotating and stationary plates. The predictions of the stability theory on the onset of convection were successfully tested. In this experiment, the finite amplitude instability in mercury for a limited range of rotation was studied. Somerville and Lipps [16] repeated Rossby's work in a three-dimensional numerical simulation. Quasi-steady and quasi-two-dimensional flows with the same experimental parameters of Rossby's were observed. Knobloch and Clune [17] focused on the previous experimental work and obtained the results of linear stability and weakly nonlinear calculations under the same experimental conditions.

In this work, a solenoidal spectral representation for the flow field is used in a Galerkin approach. As a consequence, the incompressibility (divergence free) and boundary conditions on the flow field are strictly enforced. For the sensitive nature of the parametric study undertaken, this facilitates the accurate determination of the transitory dynamic picture of the flow. The incompressibility condition appears as a constraint in the governing system of equations and is an important source of difficulty in numerical simulations. There are schemes developed solely to satisfy the continuity constraints such as the fractional step scheme by Orzag and Kells [18] and the scheme by Kleiser and Schumann [19]. However, this can only be achieved to a limited degree of accuracy. Another issue is the numerical handling of the pressure variable that usually comes without any boundary conditions. In the current approach, the pressure term is eliminated in the Galerkin projection procedure onto a solenoidal dual space.

There have been various works utilizing solenoidal spectral expansions. Moser et al. [20] presented a spectral method to automatically satisfy the continuity equation and boundary conditions and tested their method on the channel flow and the flow between concentric cylinders. They expanded the vertical and horizontal extents with Chebyshev polynomials and Fourier series, respectively. Kessler [21] studied steady and oscillatory regimes of Rayleigh-Bénard convection with explicitly constructed solenoidal bases based on poloidaltoroidal decomposition. Trigonometric polynomials and the beam functions were used in the construction of the solenoidal bases satisfying the boundary conditions in a rectangular container. Gelfgat [22] carried out a parametric study for Rayleigh-Bénard convection in rectangular 2D and 3D boxes with divergence-free Galerkin method based on Chebyshev polynomials of the first and second types. Clever and Busse [12] used toroidal-poloidal expansion in their numerical approach satisfying the solenoidal condition exactly; however, the procedure for eliminating the pressure leads to higher order differentials. Puigjaner et al. [23] studied numerically stability and bifurcation in convective flow in air in a cubical cavity heated from below. They used a divergence-free Galerkin spectral method to discretize the system and a parameter continuation method to determine the different branches of solution. They used combination of trigonometric and hyperbolic functions instead of Jacobi polynomial family. Most recently Meseguer and Trefethen [24] proposed a spectral Petrov-Galerkin formulation based on divergence-free bases in terms of Chebyshev polynomials to study stability of pipe flow.

In this work, the application of solenoidal spectral Galerkin approach to thermal convection under rotation utilizes Legendre polynomials as the underlying spectral representation. The use of Legendre polynomials avails GaussLegendre-Lobatto quadrature integration for the accurate evaluation of the inner product integrals resulting from Galerkin projection onto the dual space. The dual space is spanned by dual bases that are also required to satisfy the solenoidal condition in a form that incorporates the associated weight arising from the inner product in the spectral representation. This is required in order to eliminate the pressure term in the projection procedure. However, the use of Legendre polynomials significantly simplifies the construction of the solenoidal dual bases due to associated unity weight.

\section{Governing Equations}

Boussinesq equations are used as a model of buoyancy driven thermal convection with a coriolis term arising due to rotation in a physical domain between rotating rigid plates as illustrated in Figure 1. Under the scaling of the respective physical variables based on the thermal diffusion time $h^{2} / \alpha$, the fluid layer half depth $h=H / 2$, temperature difference between the rigid plates $\Delta T$, and the dimensionless form of the equations take the form:

$$
\nabla \cdot \mathbf{u}=0
$$

$$
\begin{aligned}
\frac{\partial \mathbf{u}}{\partial t}+ & (\mathbf{u} \cdot \nabla) \mathbf{u} \\
& =-\nabla p+\operatorname{PrRa} \theta \mathbf{e}_{z}+\operatorname{Pr} \nabla^{2} \mathbf{u}-2 \operatorname{Pr} \Omega \mathbf{e}_{z} \times \mathbf{u},
\end{aligned}
$$




$$
\frac{\partial \theta}{\partial t}+(\mathbf{u} \cdot \nabla) \theta=\frac{\mathbf{u} \cdot \mathbf{e}_{z}}{2}+\nabla^{2} \theta
$$

where

$$
\Omega=\frac{\Omega_{z} h^{2}}{v}, \quad \mathrm{Ra}=\frac{g \beta \Delta T h^{3}}{v \alpha}, \quad \operatorname{Pr}=\frac{v}{\alpha}
$$

are the Coriolis parameter $(\Omega)$ and Rayleigh (Ra) and Prandtl (Pr) numbers, respectively. The use of fluid layer half depth as the spatial scale, so that $-1 \leq z \leq 1$ for convenience, results in Ra and $\Omega$ based on the half depth. The square of the Coriolis parameter is known as Taylor number $\left(\mathrm{Ta}=4 \Omega^{2}\right)$. The quantities involved are $\Omega_{z}$ as the rotation rate about the vertical axis, $\alpha$ as the thermal diffusivity, $\beta$ as the thermal expansion coefficient, $g$ as acceleration of gravity, and $v$ as the kinematic viscosity. The convective motions are described by the velocity $\mathbf{u}=(u, v, w)$ and the temperature $\theta$, which represents the deviation from the conductive profile.

The no-slip boundary conditions at the upper and lower rigid walls are considered:

$$
\mathbf{u}=\mathbf{0}, \quad \theta=0,
$$

at the rigid plates $(z= \pm 1)$ in the $z$-direction where $\mathbf{e}_{z}$ is the direction vector opposite to gravity. The flow in the infinite horizontal extent is assumed to be periodic in the $x$ - and $y$ directions. Our investigation is limited to those cases with small rotation rate so that centrifugal force is negligible in comparison with gravity [6].

\section{Numerical Procedure and the Bases}

3.1. Considerations. The assumption of periodicity allows the use of Fourier representation in the $x$ - and $y$-directions for the velocity and temperature fields in the form:

$$
\begin{gathered}
\mathbf{u}(\mathbf{x}, t)=\sum_{|m| \leq N_{x} / 2} \sum_{|n| \leq N_{y} / 2} e^{i k_{x} x+i k_{y} y} \\
\quad \times \sum_{p=0}^{Q}\left(a_{p}^{(1)} \mathbf{V}_{p}^{(1)}(z)+a_{p}^{(2)} \mathbf{V}_{p}^{(2)}(z)\right), \\
\theta(\mathbf{x}, t)=\sum_{|m| \leq N_{x} / 2} \sum_{|n| \leq N_{y} / 2} e^{i k_{x} x+i k_{y} y} \sum_{p=0}^{Q} b_{p} T_{p}(z),
\end{gathered}
$$

for the wave numbers

$$
\begin{array}{ll}
k_{x}=\frac{2 \pi n}{s_{x}}, & -\frac{N_{x}}{2} \leq n \leq \frac{N_{x}}{2}, \\
k_{y}=\frac{2 \pi m}{s_{y}}, & -\frac{N_{y}}{2} \leq n \leq \frac{N_{y}}{2},
\end{array}
$$

where $s_{x}=L_{x} / h, s_{y}=L_{y} / h$ are the scaled periods in $x$ and $y$-directions, respectively, $a_{p}^{(j)}(t), b_{p}(t)$ are the expansion coefficients, and $\left\{\mathbf{V}_{p}^{(j)}(z), T_{p}(z)\right\}$ are the set of basis functions. The set of basis functions are chosen to satisfy

$$
\nabla \cdot\left(e^{i k_{x} x+i k_{y} y} \mathbf{V}_{p}^{(j)}(z)\right)=0, \quad \mathbf{V}_{p}^{(j)}(z=\mp 1)=\mathbf{0},
$$

for $j=1,2$ and $T_{p}(z=\mp 1)=0$. The velocity basis functions $\mathbf{V}_{p}^{(j)}(z)$ come in pairs due to the fact that the continuity equation (1) reduces the degree of freedom in determining the velocity field to two by connecting the three components.

Substitution of the representations (6) and (7) into the governing (2) and (3) results in a system of ordinary differential equations for the coefficients $a_{p}^{(j)}(t), b_{p}(t)$ after a projection procedure which is carried out by the inner product

$$
(\mathbf{f}, \mathbf{g})=\int_{-1}^{1} \omega(z) \mathbf{f}^{*} \cdot \mathbf{g} d z
$$

using the orthogonality of Fourier expansion for each wavenumber pair $\left(k_{x}, k_{y}\right)$, where $\mathbf{g}$ belongs to the physical space and $\mathbf{f}$ to the dual space, $*$ denotes the conjugate transpose, and $\omega(z)$ is a suitable weight function. The projection step requires construction of a set of dual basis functions $\left\{\overline{\mathbf{V}}_{p}^{(j)}(z), \bar{T}_{p}(z)\right\}$. In the construction of the dual velocity basis functions $\overline{\mathbf{V}}_{p}^{(j)}(z)$, the key consideration is the elimination of the pressure term from the resulting equations. This is accomplished by the conditions:

$$
\nabla \cdot\left(\omega(z) e^{i k_{x} x+i k_{y} y} \overline{\mathbf{V}}_{p}^{(j)}(z)\right)=0, \quad \overline{\mathbf{V}}_{p}^{(j)}(z=\mp 1) \cdot \mathbf{n}=0,
$$

where $\mathbf{n}$ is the unit outward normal at the plates. The dual basis functions $\bar{T}_{p}(z)$ is required to satisfy $\bar{T}_{p}(z= \pm 1)=0$.

Finally, an underlying spectral representation for the basis functions based on a class of Jacobi polynomials, namely, Legendre polynomials, is utilized. There are two considerations in this choice. First, the inherent weight function $\omega(z)=1$ associated with Legendre polynomials makes the construction of the solenoidal dual bases easier. Second, the availability of the Gauss-Legendre-Lobatto (GLL) quadrature facilitates accurate evaluation of the projection integrals

$$
(\mathbf{f}, \mathbf{g})=\int_{-1}^{1} \mathbf{f}^{*} \cdot \mathbf{g} d z \approx \sum_{j=0}^{N_{z}} \omega_{j} \mathbf{f}^{*}\left(z_{j}\right) \cdot \mathbf{g}^{*}\left(z_{j}\right),
$$

for the integrands of order $2 N_{z}-1$. Further, the denser distribution of the GLL nodes near the boundaries of the plates provides the desired resolution for the boundary layers.

3.2. Construction. The solenoidal condition (1) in Fourier representation becomes

$$
i k_{x} \widehat{u}(m, n, z, t)+i k_{y} \widehat{v}(m, n, z, t)+\mathbb{D} \widehat{w}(m, n, z, t)=0 .
$$

Here, $\mathbb{D}^{2}=d^{2} / d z^{2}$ stands for a part of the Laplacian $\nabla^{2}$. This provides the main constraint for the construction of the solenoidal and dual bases. The degree of freedom in the representation of a solenoidal flow field is reduced to two as the three components of the velocity field are connected through (13). Solenoidal nature of the flow field also 
involves the usual toroidal-poloidal decomposition of the motion. Therefore, first bases, $\mathbf{V}_{p}^{(1)}$, are lacking in their vertical velocity components while the second bases, $\mathbf{V}_{p}^{(2)}$, are lacking in their vertical vorticity components. According to this classification, the expansion coefficient $a_{p}^{(1)}$ is associated with the toroidal component and $a_{p}^{(2)}$ is associated with the poloidal component of the velocity field. The subspace $S$ of the solenoidal flow field and the dual subspace $D$ as constrained by (9), (11), and (13) are constructed as follows.

Case 1. $k_{x} \neq 0$ and/or $k_{y} \neq 0$ :

$$
\begin{gathered}
S=\operatorname{span}\left\{\left[\begin{array}{c}
-\left(\frac{k_{y}}{k_{x}}\right) g(z) \\
g(z) \\
0
\end{array}\right],\left[\begin{array}{c}
i k_{x} \mathbb{D} h(z) \\
i k_{y} \mathbb{D} h(z) \\
\left(k_{x}^{2}+k_{y}^{2}\right) h(z)
\end{array}\right]\right\}, \\
D=\operatorname{span}\left\{\left[\begin{array}{c}
-\left(\frac{k_{y}}{k_{x}}\right) f(z) \\
f(z) \\
0
\end{array}\right],\left[\begin{array}{c}
i k_{x} \mathbb{D} g(z) \\
i k_{y} \mathbb{D} g(z) \\
\left(k_{x}^{2}+k_{y}^{2}\right) g(z)
\end{array}\right]\right\} .
\end{gathered}
$$

Case 2. $k_{x}=0$ and $k_{y}=0$ :

$$
\begin{gathered}
S=\operatorname{span}\left\{\left[\begin{array}{c}
-g(z) \\
0 \\
0
\end{array}\right],\left[\begin{array}{c}
0 \\
g(z) \\
0
\end{array}\right]\right\}, \\
D=\operatorname{span}\left\{\left[\begin{array}{c}
-f(z) \\
0 \\
0
\end{array}\right],\left[\begin{array}{c}
0 \\
f(z) \\
0
\end{array}\right]\right\}, \\
T_{p}(z)=\bar{T}_{p}(z)=g(z),
\end{gathered}
$$

where $f(z)=L_{p}(z), g(z)=\left(1-z^{2}\right) L_{p}(z)$, and $h(z)=$ $\left(1-z^{2}\right)^{2} L_{p}(z)$ are selected to satisfy the boundary conditions, and $L_{p}(z)$ is the Legendre polynomial of order $p$. Besides satisfying the solenoidal and boundary conditions, these forms also satisfy

$$
\begin{gathered}
\int_{-1}^{1} \overline{\mathbf{V}}_{q}^{(1)^{*}} \cdot \mathbf{V}_{p}^{(2)} d z=\int_{-1}^{1} \overline{\mathbf{V}}_{q}^{(2)^{*}} \cdot \mathbf{V}_{p}^{(1)}, \quad d z=0, \\
\int_{-1}^{1} \overline{\mathbf{V}}_{q}^{(1)^{*}} \cdot \mathbb{D}^{2} \mathbf{V}_{p}^{(2)} d z=\int_{-1}^{1} \overline{\mathbf{V}}_{q}^{(2)^{*}} \cdot \mathbb{D}^{2} \mathbf{V}_{p}^{(1)}, \quad d z=0 .
\end{gathered}
$$

that result in some desirable decoupling features in the resulting system of equations. It can be shown that, associated with these forms, the number of quadrature nodes $N_{z}$ and the number of solenoidal bases $Q$ should be related in the least by $N_{z}=Q+4$ for the linear case and $N_{z}=3 / 2 Q+5$ for the nonlinear case to render the numerical quadrature exact in the inner product integrals.

\section{Numerical Implementation}

When a representation of the solenoidal flow field in the form of (6) and (7) is substituted into the governing equations (2) and (3), the residuals arise:

$$
\begin{aligned}
R_{\mathbf{u}}= & -\frac{\partial \mathbf{u}}{\partial t}-(\mathbf{u} \cdot \nabla) \mathbf{u}-\nabla p+\operatorname{PrRa} \theta \mathbf{e}_{z} \\
& +\operatorname{Pr} \nabla^{2} \mathbf{u}-2 \operatorname{Pr} \Omega \mathbf{e}_{z} \times \mathbf{u}, \\
R_{\theta}= & -\frac{\partial \theta}{\partial t}-(\mathbf{u} \cdot \nabla) \theta-\frac{\mathbf{u} \cdot \mathbf{e}_{z}}{2}+\nabla^{2} \theta .
\end{aligned}
$$

The projection of the residual onto the dual space spanned by $\overline{\mathbf{V}}_{p}^{(1)}, \overline{\mathbf{V}}_{p}^{(2)}$, and $\bar{T}_{p}$ is annulled,

$$
\left(\overline{\mathbf{V}}, R_{\mathbf{u}}\right)=0, \quad\left(\bar{T}, R_{\theta}\right)=0,
$$

in the Galerkin procedure to yield the weak form:

$$
\begin{aligned}
& \underbrace{\left(\begin{array}{ccc}
\left(\overline{\mathbf{V}}^{(1)}, \widehat{\mathbf{V}}^{(1)}\right) & 0 & 0 \\
0 & \left(\overline{\mathbf{V}}^{(2)}, \widehat{\mathbf{V}}^{(2)}\right) & 0 \\
0 & (\bar{T}, \widehat{T})
\end{array}\right)}_{\mathbf{A}} \\
& \times\left(\begin{array}{c}
\dot{a}^{(1)} \\
\dot{a}^{(2)} \\
\dot{b}
\end{array}\right)+\left(\begin{array}{c}
c^{(1)} \\
c^{(2)} \\
d
\end{array}\right) \\
& =\underbrace{\operatorname{Pr}\left(\begin{array}{ccc}
\left(\overline{\mathbf{V}}^{(1)},\left(\nabla^{2}-2 \Omega e_{z}\right) \widehat{\mathbf{V}}^{(1)}\right) & \left(\overline{\mathbf{V}}^{(1)},-2 \Omega e_{z} \widehat{\mathbf{V}}^{(2)}\right) & 0 \\
\left(\overline{\mathbf{V}}^{(2)},-2 \Omega e_{z} \widehat{\mathbf{V}}^{(1)}\right) & \left(\overline{\mathbf{V}}^{(2)},\left(\nabla^{2}-2 \Omega e_{z}\right) \widehat{\mathbf{V}}^{(2)}\right) & \operatorname{Ra}\left(\overline{\mathbf{V}}^{(2)}, \widehat{T} e_{z}\right) \\
0 & \frac{\left(\bar{T}, \widehat{\mathbf{V}}^{(2)} \cdot e_{z}\right)}{2 \operatorname{Pr}} & \frac{\left(\bar{T}, \nabla^{2} \widehat{T}\right)}{\operatorname{Pr}}
\end{array}\right)}_{\mathbf{B}} \\
& \times\left(\begin{array}{c}
a^{(1)} \\
a^{(2)} \\
b
\end{array}\right),
\end{aligned}
$$

where $a^{(1)}, a^{(2)}$, and $b$ are the time dependent expansion coefficients, and $c^{(1)}, c^{(2)}$, and $d$ are the projections of the nonlinear terms.

4.1. Linear Stability Analysis. The solenoidal bases and the projection procedure are tested on the linear stability of the conductive (no-motion) state leading to the critical values when the convective motion just sets in. This amounts to elimination of the terms $c^{(1)}, c^{(2)}$, and $d$ in (19). The resulting linear system of ordinary differential equations reduces to a generalized eigenvalue problem

$$
\lambda \mathrm{AE}=\mathrm{BE},
$$

for the eigenvalues $\lambda$ when a time dependence in the form

$$
\left[a^{(1)} ; a^{(2)} ; b\right]=\mathbf{E} e^{\lambda t}
$$


is assumed. As the rightmost eigenvalue in the complex plane crosses the imaginary axis as Rayleigh number is varied for a given wavenumber pair $\left(k_{x}=2 \pi / s_{x}, k_{y}=0\right)$ or $\left(k_{x}=\right.$ $\left.0, k_{y}=2 \pi / s_{y}\right)$, the system becomes unstable to infinitesimal perturbations. The resulting critical Rayleigh number $\mathrm{Ra}_{c}$ and critical wavenumber $k_{c}$ values form the marginal stability curve.

4.2. Nonlinear Regime. The computation of nonlinear terms consumes more time in Fourier-Legendre space than in real space. Thus, all nonlinear terms are computed in real space and then projected onto Fourier-Legendre space to obtain the projections of the nonlinear terms

$$
c^{1,2}=\left(\overline{\mathbf{V}}^{(1,2)},(\mathbf{u} \cdot \nabla) \mathbf{u}\right), \quad d=(\bar{T},(\mathbf{u} \cdot \nabla) \theta) .
$$

The derivatives of velocity and temperature fields in horizontal directions are calculated basically by the Fast Fourier transform (FFT), while polynomial differentiation matrix based on GLL collocation points is used for the vertical direction. The details and the associated MATLAB files for the polynomial differentiation can be found in [25].

For time discretization, semi-implicit numerical integration is used. The nonlinear and driving (buoyancy) terms are integrated explicitly using the third-order Adams-Bashforth scheme, while diffusive terms are integrated implicitly by Adams-Moulton scheme [26].

\section{Results}

A Fortran code is developed for the implementation of the proposed numerical procedure. It is tested for possible bugs, and resolution independence is constantly monitored. A linear stability analysis is conducted first, and the results are compared with the literature. The critical wavenumber $\left(k_{c}\right)$ and Rayleigh number $\left(\mathrm{Ra}_{c}\right)$ values are listed in Table 1 for the rightmost eigenvalue just crossing the imaginary axis. Some most critical values, $\mathrm{Ra}_{c}^{*}$ and $k_{c}^{*}$, corresponding to the least $\mathrm{Ra}_{c}$ value on each marginal stability curve as indicated by “+” markers in Figure 2, are shown in Table 2. These are obtained by the selection of $(n=0, m=1)$ (or equivalently $(n=$ $1, m=0)$ due to degeneracy in the horizontal directions) as defined in (8). They are in agreement with the known linear stability results for the rotating Rayleigh-Bénard problem. In addition, the most critical wavenumber and the corresponding Rayleigh number variation with rotation, namely, $k_{c}^{*}(\Omega)$ and $\operatorname{Ra}_{c}^{*}(\Omega)$, are presented in Figures 3 and 4, respectively. There is a good agreement between the computed critical values and the literature $[5,6,27]$. Linear stability analysis can be performed with high accuracy by means of the proposed method. While typical runs for the linear stability calculations take a few CPU seconds, nonlinear simulations require higher $\mathrm{CPU}$ time because of time integrations and Fourier-Legendre transforms to be performed in high spatial resolutions. For example, typical computing time is approximately 3 hours for integrating 100 nondimensional seconds a long with $N_{x} \times N_{y} \times N_{z}=16 \times 16 \times 20$ resolution in $3.0 \mathrm{GHz}$ CPU unit.

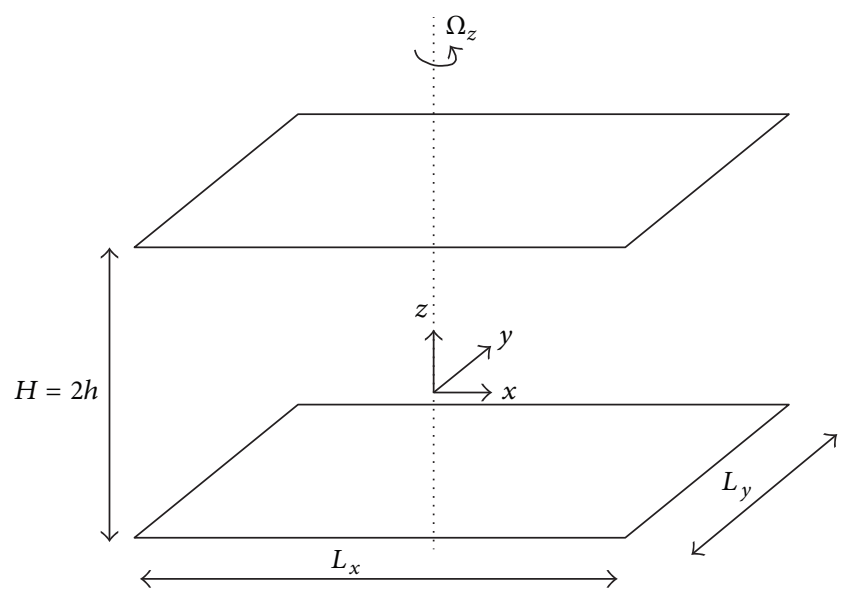

FIGURE 1: Convective box influenced by rotation.

Nonlinear simulations are performed for varying control parameters, and the Nusselt number is computed. It is observed in Figure 5(c) that limited rotation in moderate Prandtl fluids stabilizes the convection and suppresses the oscillations in high Rayleigh number flow. This phenomenon is also observed in [9]. Increased heat flux is caused by the stabilizing effect of limited rotation in the range $\Omega=0-30$. Coriolis force can balance horizontal temperature gradients, and, hence, less potential energy is released by horizontal temperature gradients. On the other hand, increasing rotation rate destabilizes the system and introduces new oscillatory motions for $\mathrm{Ra}-\mathrm{Ra}_{c}=20000$ [12]. Increased heat flux with limited rotation is also observed for low Rayleigh number flows in Figure 5((a) and (b)), but this increment is very low because convection is still two-dimensional at these parameter values. Heat flux starts decreasing at $\Omega=10$ for $\mathrm{Ra}-\mathrm{Ra}_{c}=2000, \Omega=30$ for $\mathrm{Ra}-\mathrm{Ra}_{c}=10000$, and $\Omega=30$ for $\mathrm{Ra}-\mathrm{Ra}_{c}=20000$ because horizontal velocities destabilize the system with increasing rotation. In [9], it is indicated that rotational constraint balances the nonlinear processes for up to $\mathrm{Ta} \leq 10^{3.6}(\Omega \approx 31)$ for convection between free boundaries. More recent work conducted by [12] reported that rolls are unstable for $\operatorname{Pr}>1$ beyond $\Omega \approx 27$.

Coriolis term depends linearly on the horizontal velocity. Hence, increasing rotation rate magnifies the toroidal energy (that is associated with $\mathbf{V}_{p}^{(1)}$ component of the solenoidal flow field) as shown in Figure 6. For all Rayleigh number values, increasing rotation absorbs the poloidal energy and stimulates the toroidal energy. Increase in toroidal energy is very rapid for $\mathrm{Ra}-\mathrm{Ra}_{c}=2000$ and $\mathrm{Ra}-\mathrm{Ra}_{c}=10000$ because both cases correspond to steady two-dimensional convection regime in nonrotating system. Limited Coriolis force, for example $\Omega=10$, does not affect the roll structure but introduces three-dimensional motions as it is seen in Figures 7 and 8. This motion appears in oblique angle to the roll direction as also observed by [8]. On the other hand, $\mathrm{Ra}-$ $\mathrm{Ra}_{c}=20000$ case exhibits a different behavior. Since it corresponds to periodic motion regime in nonrotating system, 
TABLE 1: Critical wavenumber and rayleigh number values at which convection just sets in $(\Omega=0)$.

\begin{tabular}{lcccccccc}
\hline$k_{c}$ & 1 & 2 & 3 & 3.117 & 4 & 5 & 7 & 6 \\
$\mathrm{Ra}_{c}$ & 5854.48 & 2177.41 & 1711.27 & 1707.76 & 1879.25 & 2439.32 & 3417.98 & 4918.54 \\
\hline
\end{tabular}

TABle 2: Comparison of the most critical values with Chandrasekhar's calculation [5] at $8 \times 8 \times 8$ resolution.

\begin{tabular}{lcccc}
\hline$\Omega\left(\mathrm{Ta}=4 \Omega^{2}\right)$ & $\mathrm{Ra}_{c}^{*}$ & $\mathrm{Ra}_{c}^{*}[5]$ & $k_{c}^{*}$ & $k_{c}^{*}[5]$ \\
\hline 5 & 1756.34 & 1756.6 & 3.16 & 3.15 \\
50 & 4712.04 & 4713.1 & 4.78 & 4.80 \\
500 & 70843.90 & 71132 & 10.79 & 10.80 \\
\hline
\end{tabular}

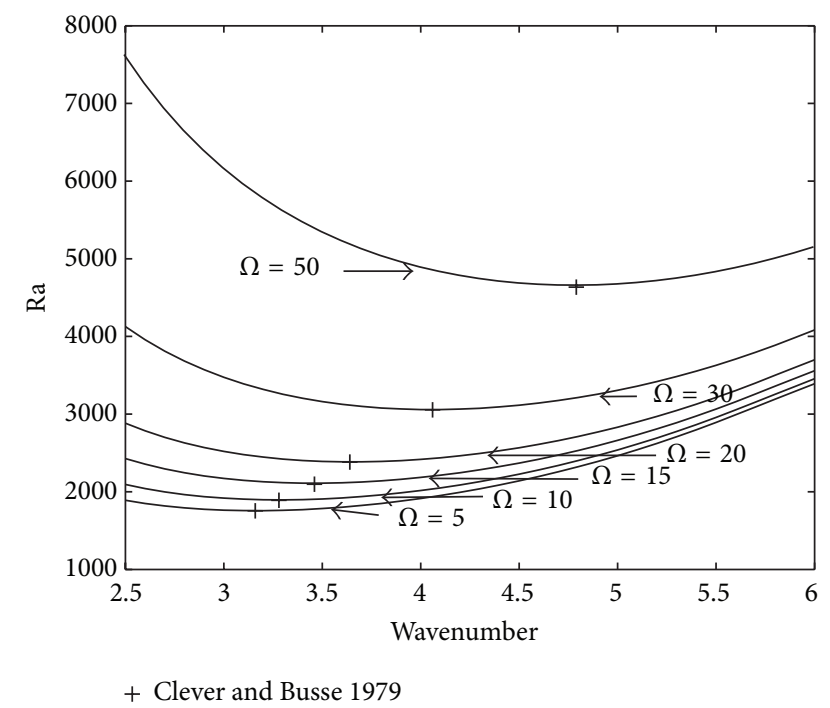

FIGURE 2: Marginal stability curves as effected by different rotation rates.

it contains small-scale motions and vertical vorticity component. The motion tends towards two-dimensional convection with increasing rotation and toroidal energy sharply decreases, while poloidal energy conversely increases for $\Omega \approx$ 15. It is caused by the stabilizing effect of limited rotation as explained above. This effect also increases the heat transport because of increasing poloidal component. As vertical shear increases, $\Omega \geq 15$ for low Rayleigh and $\Omega \geq 30$ for high Rayleigh numbers, toroidal motion dominates and brings unstable character.

\section{Conclusions}

In this work, solenoidal spectral approach is used to study rotating Rayleigh-Bénard convection numerically for a range of parameter values. The expansion of the velocity field in terms of the solenoidal bases and the subsequent projection onto the solenoidal dual space provide the automatic satisfaction of the divergence-free condition and the elimination of the pressure. Removal of these common algorithmic difficulties helps to focus on the extraction of the dynamics hidden within the model dynamical system equations.

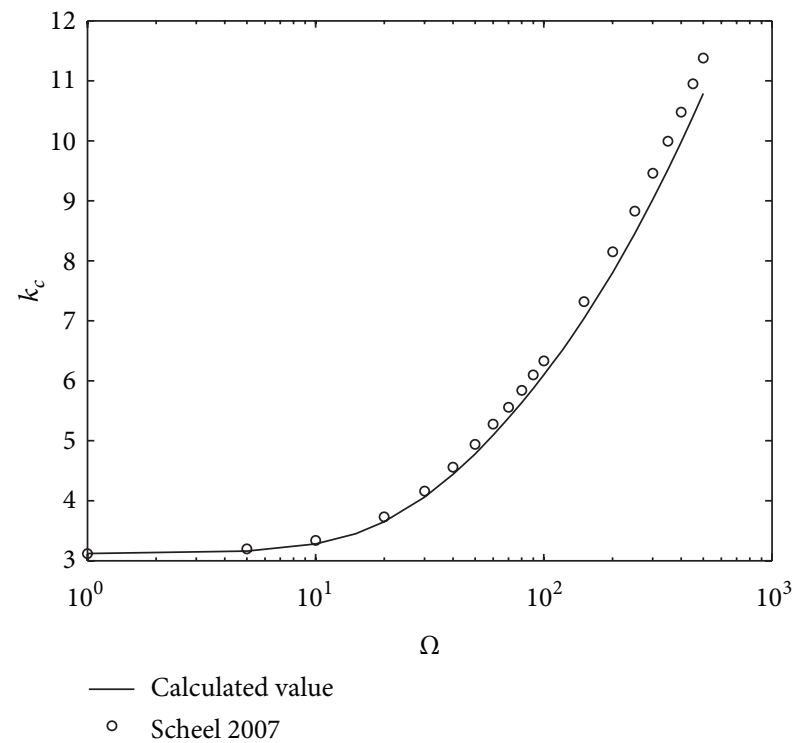

FIGURE 3: The critical wavenumber $\left(k_{c}^{*}\right)$ variation with rotation: $k_{c}^{*}(\Omega)$.

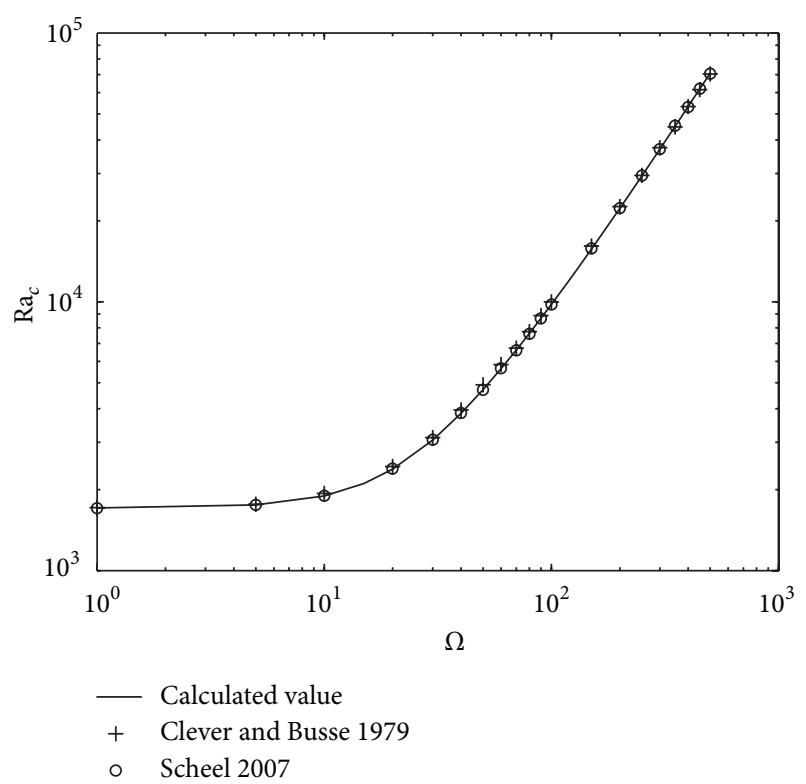

FIGURE 4: The critical Rayleigh number $\left(\mathrm{Ra}_{c}^{*}\right)$ variation with rotation: $\operatorname{Ra}_{c}^{*}(\Omega)$.

The current formulation provides a robust numerical tool to seamlessly investigate the linear and nonlinear regimes in rotating Rayleigh-Bénard convection. Form of the resulting dynamical system facilitates the use of numerical tools from 


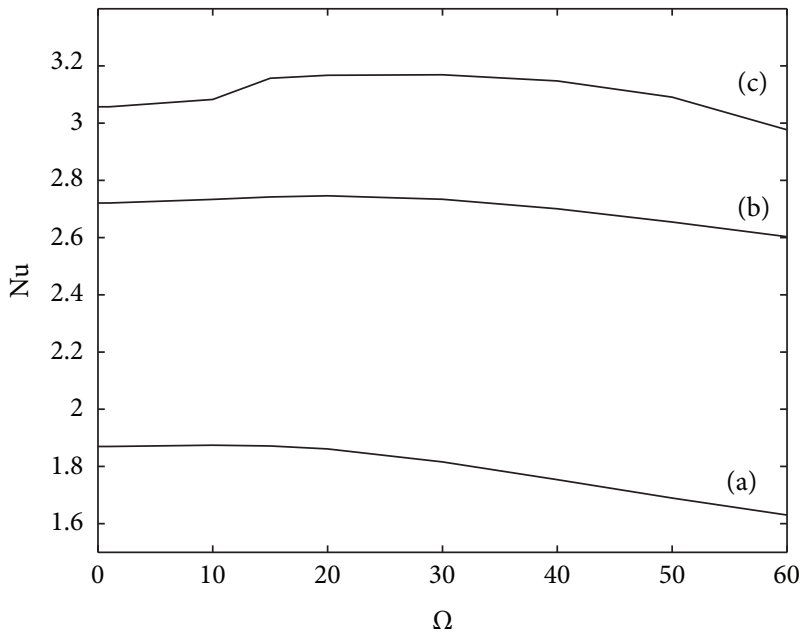

FIGURE 5: Nusselt number variation with rotation, $\mathrm{Pr}=0.71$, (a) $\mathrm{Ra}-\mathrm{Ra}_{c}=2000$, (b) $\mathrm{Ra}-\mathrm{Ra}_{c}=10000$, and (c) $\mathrm{Ra}-\mathrm{Ra}_{c}=20000$.

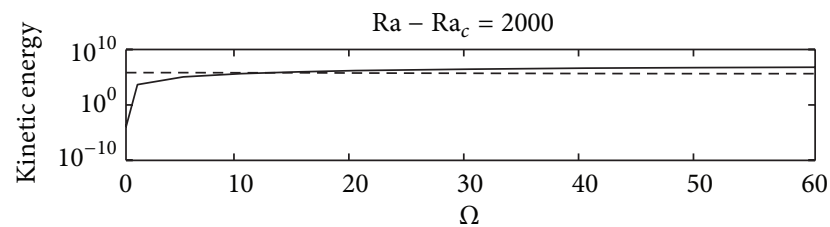

(a)

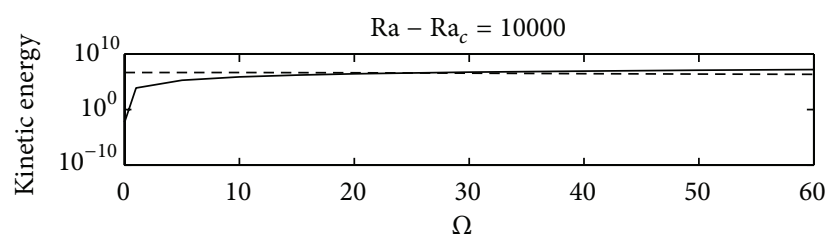

(b)

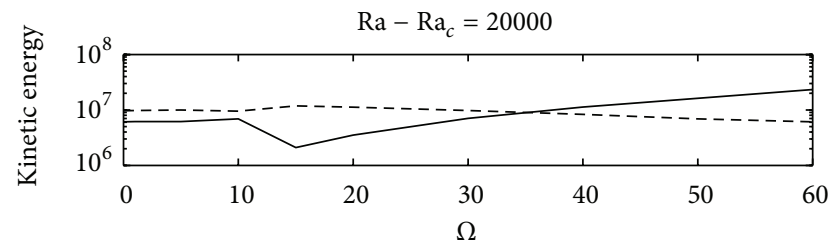

— Toroidal KE

- - - Poloidal KE

(c)

Figure 6: Toroidal and poloidal kinetic energy variation with rotation, $\mathrm{Pr}=0.71$.

the dynamical system and bifurcation theory that is a natural extension of this work.

\section{Acknowledgments}

The authors are grateful to (The Scientific and Technological Research Council of Turkey) TÜBİTAK, for providing financial support through research project 109M435 and Dr. Baran Aydin, for his suggestions.

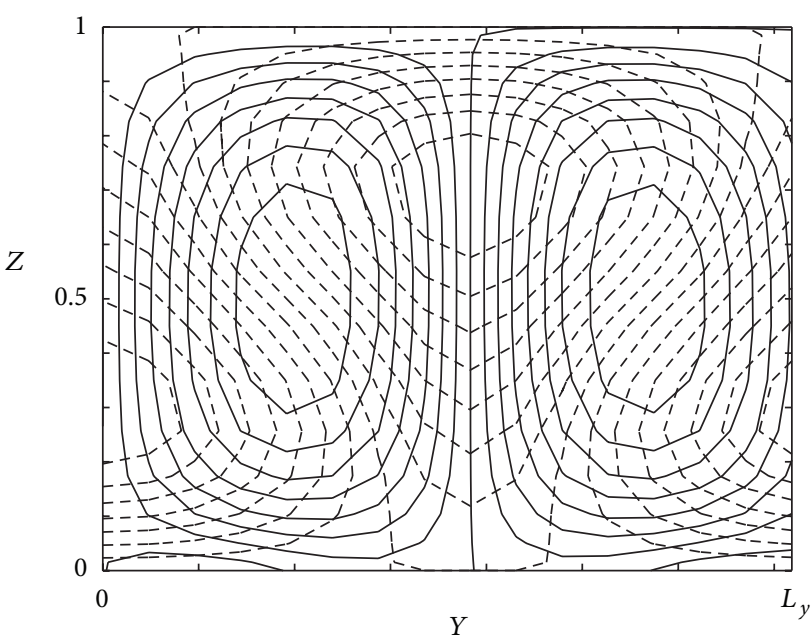

FIGURE 7: v-w streamline (solid line) and temperature contour (dash line) on $Y$ - $Z$ plane at $x=L_{x} / 4, \mathrm{Pr}=0.71, \mathrm{Ra}-\mathrm{Ra}_{c}=2000$, and $\Omega=10$.

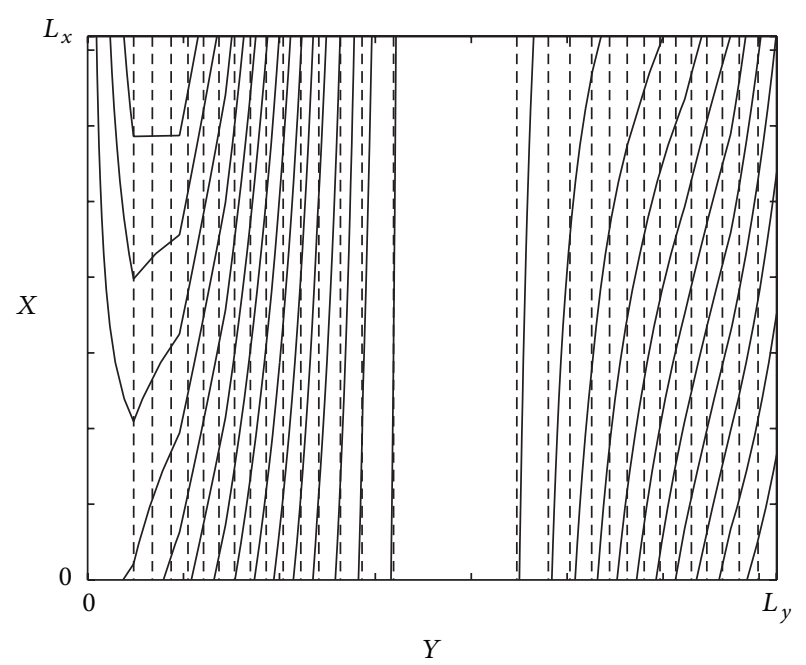

FIGURE 8: u-v streamline (solid line) and temperature contour (dash line) on $X-Y$ plane at $z=0.4861, \operatorname{Pr}=0.71$, and $\mathrm{Ra}-\mathrm{Ra}_{c}=2000$, and $\Omega=10$.

\section{References}

[1] R. Krishnamurti, "On the transition to turbulent convection. Part 1. The transition from two- to three-dimensional flow," Journal of Fluid Mechanics, vol. 42, no. 02, pp. 295-307, 1970.

[2] J. B. McLaughlin and S. A. Orszag, "Transition from periodic to chaotic thermal convection," Journal of Fluid Mechanics, vol. 122, pp. 123-142, 1982.

[3] J. P. Gollub and S. V. Benson, "Many routes to turbulent convection," Journal of Fluid Mechanics, vol. 100, no. 3, pp. 449470, 1980.

[4] O. Mikolasek, B. Barlet, E. Chia, V. Pouomogne, and E. T. M. Tomedi, "Développement de la petite pisciculture marchande au Cameroun: la recherche-action en partenariat," Cahiers Agricultures, vol. 18, no. 2-3, pp. 157-163, 2009.

[5] S. Chandrasekhar, Hydrodynamic and Hydromagnetic Stability, Clarendon Press, Oxford, UK, 1961. 
[6] R. M. Clever and F. H. Busse, "Nonlinear properties of convection rolls in a horizontal layer rotating about a vertical axis," Journal of Fluid Mechanics, vol. 94, no. 4, pp. 609-627, 1979.

[7] G. Veronis, "Motions at subcritical values of the Rayleigh number in a rotating fluid," Journal of Fluid Mechanics, vol. 24, pp. 545-554, 1966.

[8] G. Veronis, "Cellular convection with finite amplitude in a rotating fluid," Journal of Fluid Mechanics, vol. 5, pp. 401-435, 1959.

[9] G. Veronis, "Large-amplitude bénard convection in a rotating fluid," Journal of Fluid Mechanics, vol. 31, no. 01, pp. 113-139, 1968.

[10] G. Küppers and D. Lortz, “Transition from laminar convection to thermal turbulence in a rotating fluid layer," Journal of Fluid Mechanics, vol. 35, no. 3, pp. 609-620, 1969.

[11] G. Küppers, "The stability of steady finite amplitude convection in a rotating fluid layer," Physics Letters A, vol. 32, no. 1, pp. 7-8, 1970.

[12] R. M. Clever and F. H. Busse, "Convection in a low Prandtl number fluid layer rotating about a vertical axis," European Journal of Mechanics B, vol. 19, no. 2, pp. 213-227, 2000.

[13] E. Kurt, F. H. Busse, and W. Pesch, "Hydromagnetic convection in a rotating annulus with an azimuthal magnetic field," Theoretical and Computational Fluid Dynamics, vol. 18, no. 2-4, pp. 251-263, 2004.

[14] E. Kurt, W. Pesch, and F. H. Busse, "Pattern formation in the rotating cylindrical annulus with an azimuthal magnetic field at low Prandtl numbers," Journal of Vibration and Control, vol. 13, no. 9-10, pp. 1321-1330, 2007.

[15] H. T. Rossby, "A study of Bénard convection with and without rotation," Journal of Fluid Mechanics, vol. 36, no. 02, pp. 309335, 1969.

[16] R. C. J. Somerville and F. B. Lipps, "A Numerical study in three space dimensions of Benard convection in a rotating fluid," Journal of Atmospheric Sciences, vol. 30, pp. 590-596, 1973.

[17] E. Knobloch and T. Clune, "Pattern selection in rotating convection with experimental boundary conditions," Physical Review E, vol. 47, no. 4, pp. 2536-2550, 1993.

[18] S. A. Orzag and L. C. Kells, "Transition to turbulence in plane poiseuille and plane couette flow," Journal of Fluid Mechanics, vol. 96, no. 1, pp. 159-205, 1980.

[19] L. Kleiser and U. Schumann, "Treatment of incompressibility and boundary conditions in 3-D numerical spectral simulations of plane channel flows," in Proceedings of the 3rd Conference on Numerical Methods in Fluid Mechanics, E. H. Hirschel, Ed., pp. 165-173, 1980.

[20] R. D. Moser, P. Moin, and A. Leonard, "A spectral numerical method for the Navier-Stokes equations with applications to Taylor-Couette flow," Journal of Computational Physics, vol. 52, no. 3, pp. 524-544, 1983.

[21] R. Kessler, "Nonlinear transition in three-dimensional convection," Journal of Fluid Mechanics, vol. 174, pp. 357-379, 1987.

[22] A. Y. Gelfgat, "Different modes of rayleigh-bénard instability in twoand three-dimensional rectangular enclosures," Journal of Computational Physics, vol. 156, pp. 300-324, 1999.

[23] D. Puigjaner, J. Herrero, F. Giralt, and C. Simó, "Stability analysis of the flow in a cubical cavity heated from below," Physics of Fluids, vol. 16, no. 10, pp. 3639-3655, 2004.

[24] Á. Meseguer and L. N. Trefethen, "Linearized pipe flow to Reynolds number 107," Journal of Computational Physics, vol. 186, no. 1, pp. 178-197, 2003.
[25] J. A. C. Weideman and S. C. Reddy, "A MATLAB differentiation matrix suite," Association for Computing Machinery, vol. 26, no. 4, pp. 465-519, 2000.

[26] J. P. Boyd, Chebyshev and Fourier Spectral Methods, Dover, Mineola, NY, USA, 2001.

[27] J. D. Scheel, Rotating Rayleigh-Benard convection [Ph.D. thesis], California Institute of Technology, 2007. 


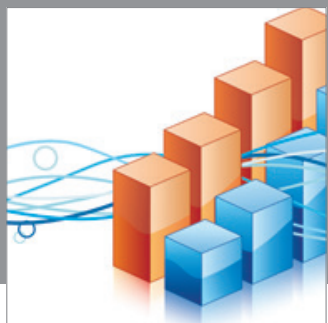

Advances in

Operations Research

mansans

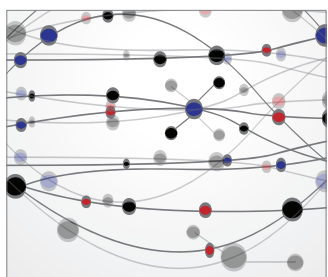

The Scientific World Journal
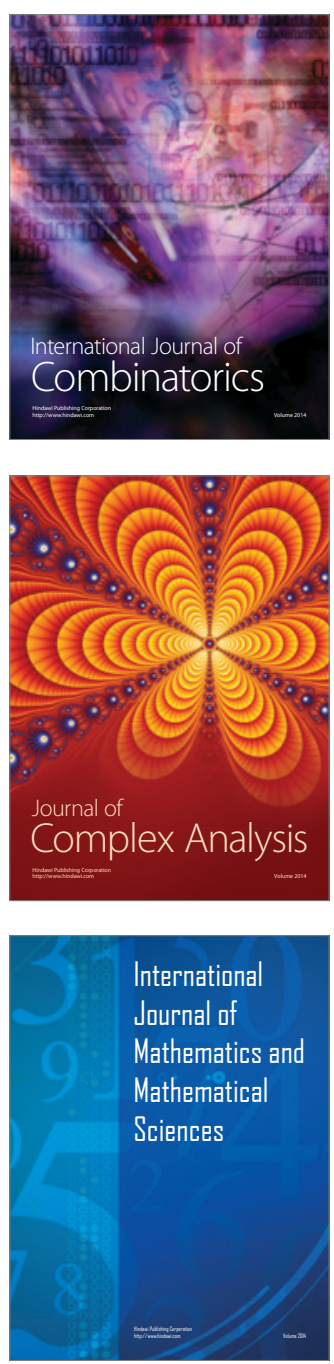
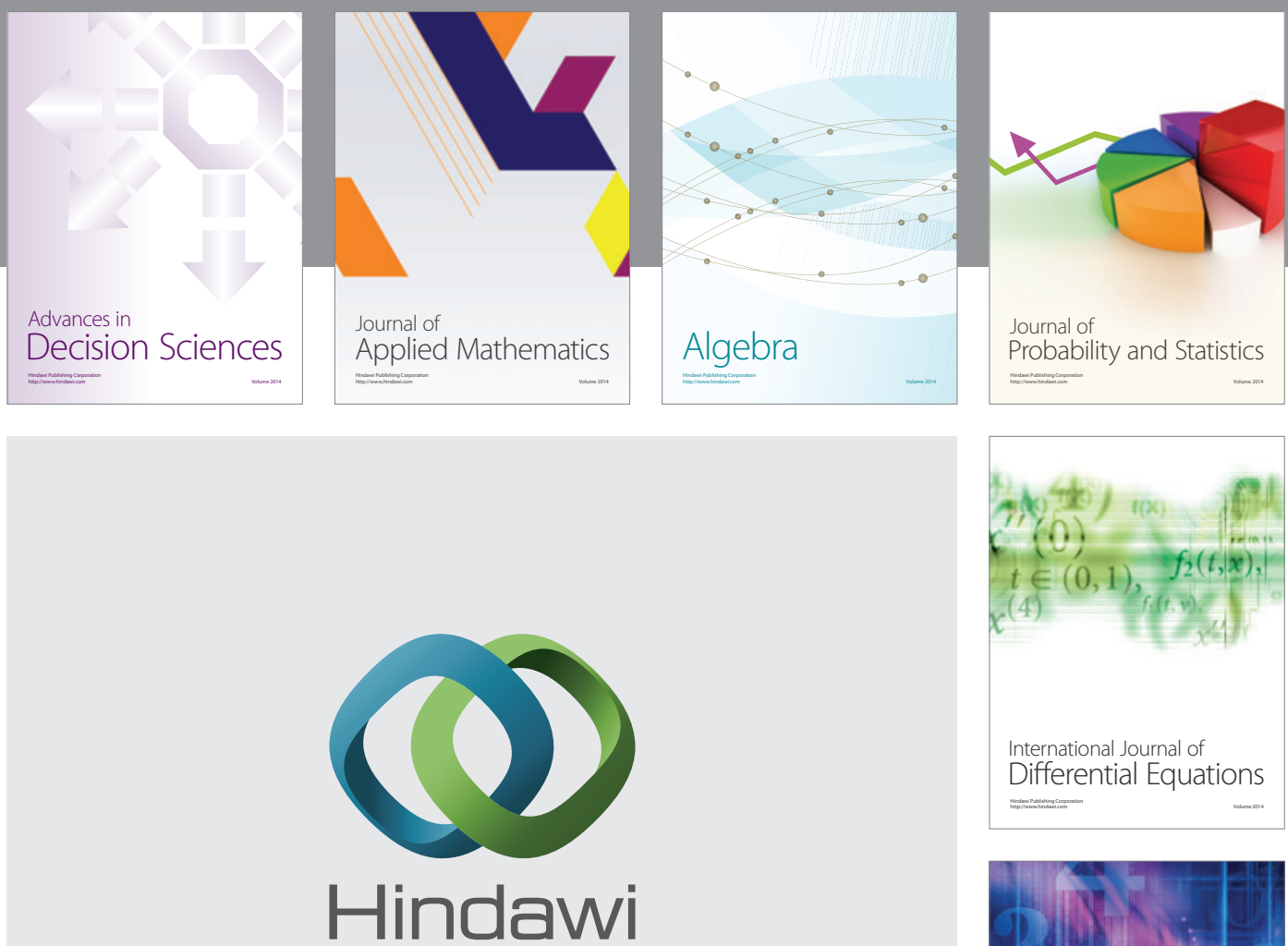

Submit your manuscripts at http://www.hindawi.com
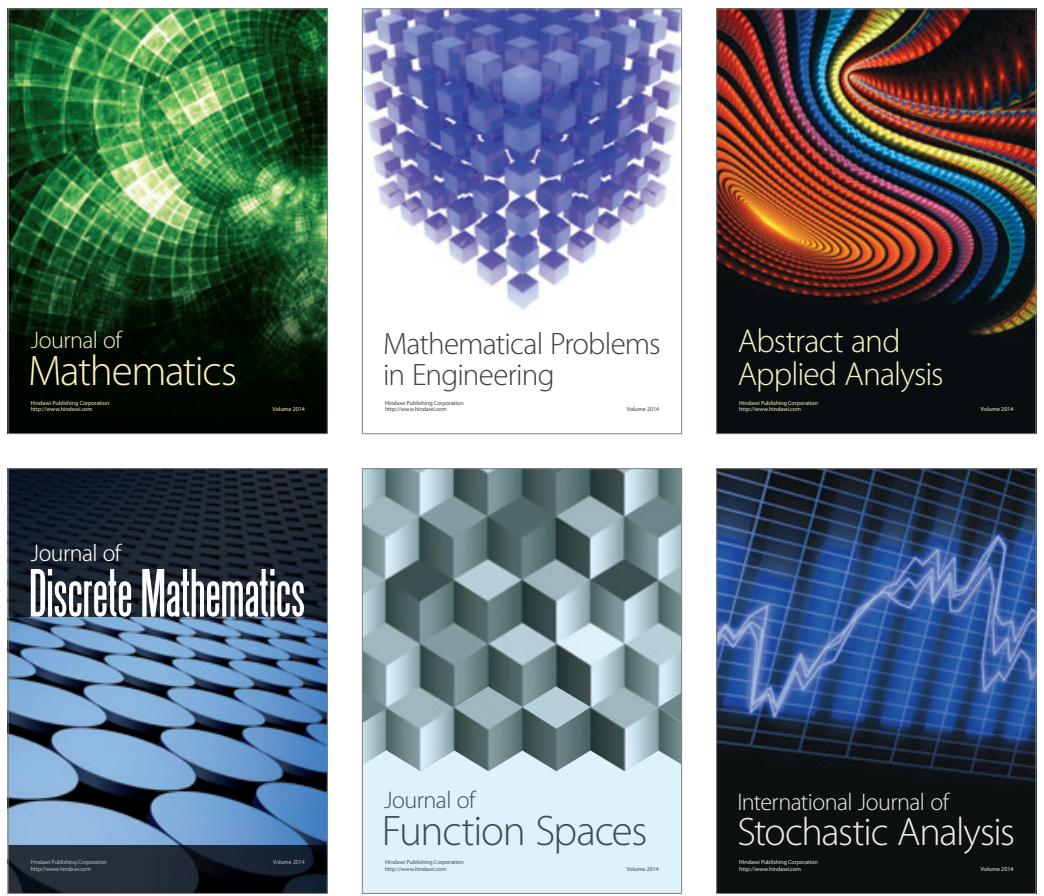

Journal of

Function Spaces

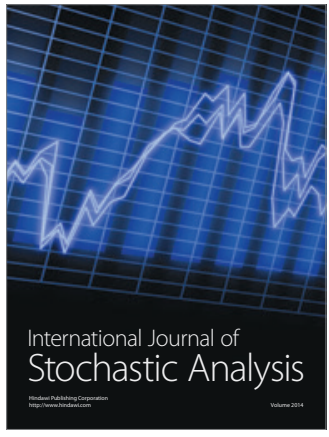

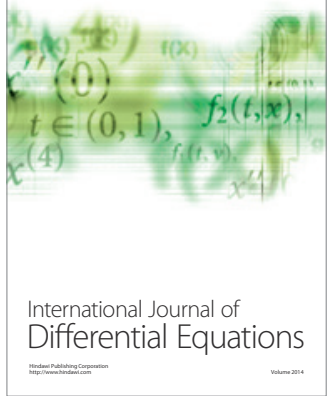
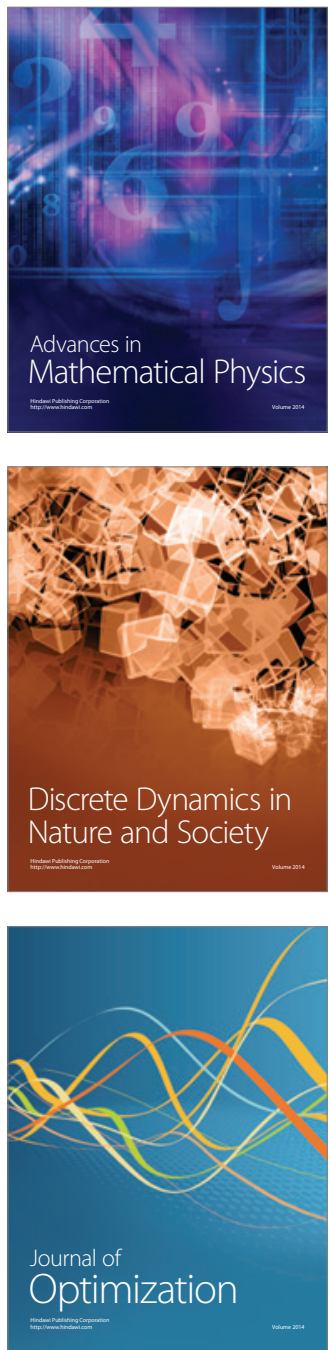